\title{
Original atide
}

\section{A profile and treatment outcome of seasonal hyper-acute panuveitis}

\author{
Shrestha E \\ Consultant Ophthalmologist, Himalaya Eye Hospital, Pokhara, Nepal
}

\begin{abstract}
Introduction: Seasonal hyper-acute panuveitis (SHAPU) is a sight-threatening disease and its management is challenging.
\end{abstract}

Objective: To study the profile and evaluate the visual outcome of the patients of clinicallydiagnosed cases of SHAPU after treatment.

Subjects and methods: A retrospective interventional hospital-based study was carried out involving 21 subjects with clinically-diagnosed SHAPU. The data were retrieved from the record section of the hospital and analyzed. The variables studied were demographic pattern, clinical condition, duration of presentation and visual acuity before and after the treatment.

Statistics: The data were analyzed using Epi Info version 2000. Percentage prevalence, mean values with standard deviation, relative risk, 95\% CI and p value were calculated. P value of < 0.05 was considered to be significant.

Results: Among the 21 cases, the numbers of male and female were 11 (52.4 \%) and 10 (46.7 $\%)$ respectively. A comparative analysis of gender in children and adults did not show any significant difference $(R R=0.47,95 \% C I=0.22-1.01$, Fisher exact test: $p=0.14)$. The mean for all ages was $7 \pm 12.68$ years, while the mean age in pediatric cases was $4.5 \pm 3.91$ years. Thirteen (61.9\%) cases occurred in children below fifteen years.

Fifteen (71.4 \%) cases reported during September and October. Presenting visual acuity of all cases was less than 3/60. All of them received medical treatment. By the end of the $4^{\text {th }}$ week, seven (33.3\%) patients regained vision to 6/18.

Conclusion: SHAPU is more prevalent in pediatric age group. It is equally prevalent among males and females. The visual acuity can improve with early medical treatment.

Keywords: SHAPU, panuveitis, steroid, phthisis bulbi

\section{Introduction}

Seasonal Hyper acute Panuveitis (SHAPU) is still a curious entity. This condition was first reported in Nepal by Malla OK (1978) as endophthalmitis probably caused by Tussock Moth. However, Upadhyay MP et al(1984)

Received: 28.08.2009 Accepted: 31.12.2010

Correspondence and reprint request to: Dr Eliya Shrestha, MD

Consultant Ophthalmologist

Himalaya Eye Hospital

Gharipatan, Pokhara.

Phone (Off)- 00977-61-461168, (Res)- 00977-61-461429

Mobile no- 9846086534

Email: eliyashrestha@yahoo.com studied it in detail and described the disease as seasonal hyper-acute panuveitis (SHAPU).

The disease has very acute presentation with redness of eyes and leukocoria accompanied by little pain. There is fibrinoid reaction in the anterior chamber and often there is a hypopyon. This is followed by hypotony and a very sudden drop in vision. Examination of the posterior segment is not possible due to leukocoria. The anterior chamber ultimately becomes flat and the eyes become pthisical (Nussenbladt \& Palestine, 1989). 
The etiology of this uveitis remains unknown. No virus or antigen has been demonstrated by serological and immunological tests. Histological study of the enucleated eye has shown the presence of chronic inflammatory cells, lymphocytes and plasma cells (Albert \& Jakobiec, 1994).

The management of this condition till date is challenging because this is a rapidly progressing disease. However, early vitrectomy has shown some success in restoring useful vision and preserving the globe. Byanju RN et al (2003) reported that early vitrectomy is a useful procedure for the treatment of SHAPU as $50 \%$ of their patients who underwent vitrectomy gained a vision of better than $6 / 60$.

Since the 1950s, when Hench at the Mayo clinic first introduced the use of cortisone in rheumatoid arthritis victims, the use of corticosteroids has been the usual practice in uveitis if no specific cause is discovered.

This study aims to find out the visual outcome in clinically-diagnosed cases of SHAPU treated with subconjunctival steroid injection and intravitreal steroid and antibiotic injections.

\section{Subjects and methods}

A retrospective interventional hospital based-study of the cases of SHAPU who attended the Himalaya Eye Hospital (HEH), Pokhara, from Janaury 2007 to March 2008 was carried out. Only the cases with a clinical picture of SHAPU were included. The diagnostic criteria set were painful red eye with rapidly progressing severe visual impairment of sudden onset, soft eye, fibrinoid anterior chamber (AC) reaction with hypopyon and USG-B scan showing exudation in the vitreous cavity.

All the patients' demographic pattern, presenting features, visual acuity, findings of slit-lamp biomicroscopy and indirect ophthalmoscopy, intraocular pressure by digital or applanation tonometry and treatment modality offered were studied. Infective endophthalmitis was ruled out clinically by the absence of history of ocular trauma or surgery and any septic focus in the body. Those with severe ocular pain following ocular trauma or surgery, congestion and chemosis were diagnosed as infective endophthalmitis and excluded from the study. Only those cases with severe vitreous exudates and vitreous haze were subjected to vitreous tap and microbiological analysis.

\section{Statistics}

The data were analyzed using Epi Info version 2000. Percentage prevalence, mean values with standard deviation, relative risk, $95 \% \mathrm{CI}$ and p value were calculated. $\mathrm{P}$ value of $<0.05$ was considered to be significant.

\section{Table 1}

Age and gender distribution comparing the gender in children and adults

\begin{tabular}{|c|c|c|c|}
\hline Age (yrs) & Female & Male & $\begin{array}{c}\text { Relative } \\
\text { risk* }\end{array}$ \\
\hline $0-5$ & 4 & 6 & \multirow{1}{*}{} \\
\hline $6-10$ & 1 & 3 & \multirow{1}{*}{0} \\
\hline $11-15$ & 1 & 1 & \multirow{2}{*}{0.47} \\
\hline $16-20$ & 1 & 1 & \\
\hline $21-25$ & 1 & 0 & \\
\hline $26-30$ & 1 & 0 & \\
\hline $51-55$ & 1 & 0 & \\
\hline Total & 10 & 11 & \\
\hline
\end{tabular}

* 95\% CI=0.22-1.01, P Value (Fisher exact test $)=0.14$

\section{Results}

A total of 21 patients were found to have clinically diagnosed SHAPU. Five cases who presented to us with severe vitreous exudates were subjected to intravitreous injection of vancomicin and amikacin along with dexamethasone.

Eleven (52.4 \%) patients were male and 10 (47.6 \%) were female, their ages ranging from 1 to 55 years. The disease was equally prevalent among males and females. A comparative analysis of gender in children and adults did not show any significant difference $(\mathrm{RR}=0.47,95 \% \mathrm{CI}=0.22-1.01$, Fisher exact test: $\mathrm{p}=$ $0.14)$. The mean for all ages was $7 \pm 12.68$ years , while the mean age in pediatric cases was $4.5 \pm 3.91$. Thirteen (61.9\%) cases occurred in children below fifteen years.

Figure 1 shows that 15 (71.4\%) out of 21 cases occurred during September and October, 2 cases each during August and November and 1 case each during December and March. No cases were reported during January and February. Only 2 (9.5 \%) cases out of 21 gave a history of contact with moths and/or being in an environment of increased population of moths especially in the evening around the source of light. 
Fig 1

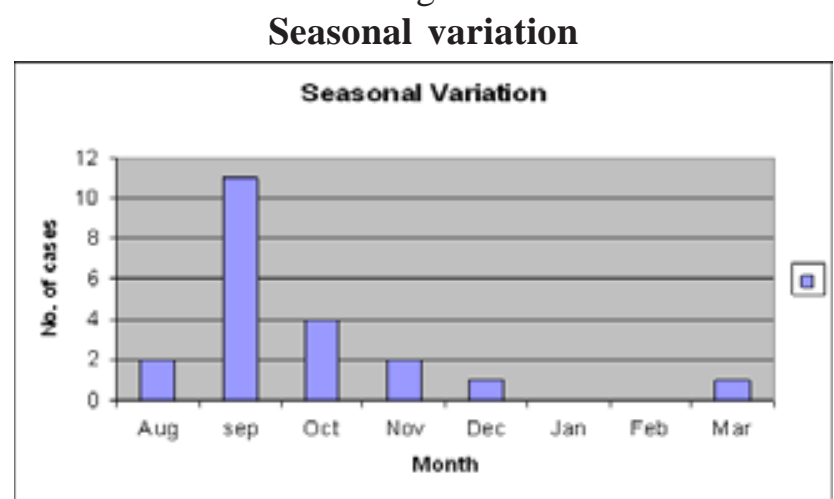

\section{Clinical manifestations}

All the cases had leukocoria, fibrinous exudates and a shallow anterior chamber. Absence of congestion was noted in $3(14.3 \%)$ cases probably due to delayed presentation.

Presenting visual acuity was less than $3 / 60$ in all the cases. Out of that, 12 (57.2\%) cases had perception of light (PL), 1 (4.7\%) had no PL, 2 (9.6 \%) had hand movements (HM), 5 (23.8\%) had counting finger close to face (CFCF) and 1 (4.7 \%) had 1/60 of vision. By the end of the $4^{\text {th }}$ week, 7 (33.3 \%) patients regained a vision of 6/18.

Of 9 patients, who regained the visual acuity of $6 / 18$ or better, 5 presented within 24 hours ( $R R=3.13$, $95 \%$ $\mathrm{CI}=1.25-7.78), \mathrm{p}=0.046)$. The vision of $5(23.8 \%)$ patients, who presented after 7 days of symptoms, could not be saved.

As table 2 shows, 13 cases were treated with subconjunctivals, 5 cases with intravitreals, 2 cases with both subconjunctival and intravitreals and one with both topical and oral steroids. Nine cases could retain a vision of $6 / 6-6 / 18$. More than $50 \%$ of the cases who underwent subconjunctival steroid injection regained a vision of $6 / 18$ or better. This was, however, not statistically significant $(\mathrm{RR}=2.15,95 \% \mathrm{CI}=0.59$ 7.91, Fisher exact test: $\mathrm{p}=0.36$ ). The subconjunctival steroid injection of dexamethasone $2 \mathrm{mg}$ was given once daily for at least one week or more than that if improvement was noticed.

\section{Discussion}

In 1978, Malla OK reported an epidemic of 13 cases of this blinding eye disease from Pokhara, Nepal for the first time. He reported it as endophthalmitis. The epidemic of this disease was in the late monsoon season
Fig 2

Early presentation versus visual outcome

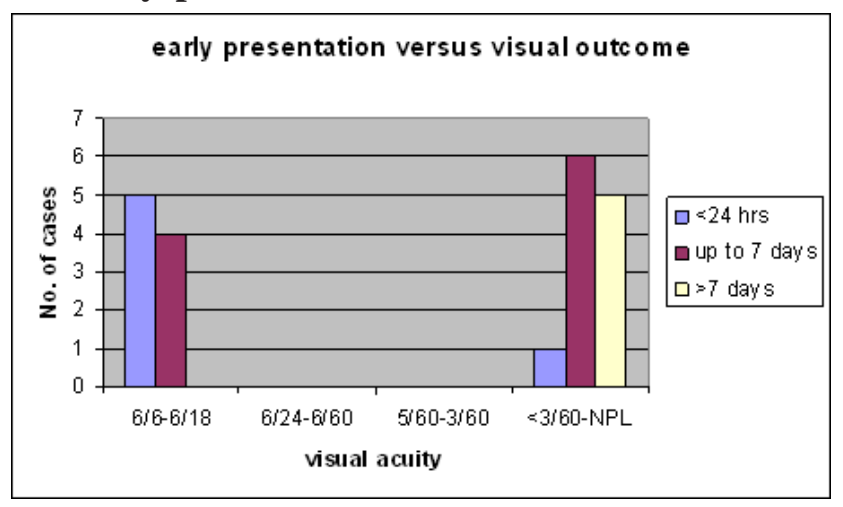

Table 2

Management versus final visual outcome

\begin{tabular}{|c|c|c|c|}
\hline \multirow{2}{*}{ Treatment option } & \multicolumn{3}{|c|}{ Final visual outcome } \\
\cline { 2 - 4 } & $6-6 / 18$ & $\begin{array}{c}6 / 18- \\
6 / 60\end{array}$ & $\begin{array}{c}<3 / 60- \\
\text { NPL }\end{array}$ \\
\hline Subconjunctival & 7 & - & 6 \\
\hline Intravitreal & 1 & - & 4 \\
\hline $\begin{array}{c}\text { Subconjunctival } \\
\text { + intravitreal, } \\
\text { both }\end{array}$ & - & - & 2 \\
\hline Topical +oral & 1 & - & - \\
\hline
\end{tabular}

(Aug to Sept 1977) which coincided with increase in the population of the Tussock moth. In his series of 13 cases, 7 were female and 6 male, with the age ranging from 3 months to 39 years. In all of them, only one eye was involved and all the eyes were blind in a week or so in spite of the earliest and the most intensive treatment. Two patients gave a definite history of contact with moth and in two others there was a suspicion of some such contact. Later in 1984, Upadhyay et al reported a similar disease occurring in two cycles after first being recognized soon after the monsoon season and named the disease as seasonal hyper-acute panuveitis (SHAPU) instead of endophthalmitis reported earlier by Malla OK (1978). They also noted that several children had been exposed to the moths, but they were unable to define if antigens from the moth had induced the uveitis. The histopathological study of the enucleated eyes revealed severe inflammatory response with lymphocytes and plasma cells with the retina drawn up into a retrolenticular mass. 
The reason for the highest occurrence of SHAPU during September and October is still unknown. Investigations on a variety of moths prevalent during the peak incidence period have failed to reproduce the disease in experimental animals (Whitcup, 2004). Byanju et al (2003) reported the disease in a similar period of the year. They noted the occurance of the epidemic in alternate years. The moth population was high during that period and all of their patients gave history of being close to the moth population found especially in the evening and around the source of light. As the disease epidemic coincides with an increase in the moth population and all of their patients gave history of contact with moths, they suggested the association of the disease with moths. Because of the intense intraocular inflammation leading rapidly to hypotony and pthisis bulbi, they felt the disease is more likely to be endophthalmitis rather than panuveitis. In contrast to the earlier findings where no eyes were saved with medical treatment, Byanju et al (2003) reported that $50 \%$ (6 out of 12) of the patients who underwent vitrectomy gained a vision of better than $6 / 60$. They reported that 2 of their patients regained visual acuity better than 6/18 with medical treatment which was probably due to early presentation and prompt treatment. They concluded that vitrectomy was a useful procedure for treatment of SHAPU.

Though none of our patients underwent vitrectomy, 9 (42.8\%) cases regained a vision of $6 / 18$ or better. Of them 7 cases were given sub-conjunctival steroid injections along with topical medicines, 1 was treated with intravitreal injection and 1 case was treated with topical and oral steroids. This is probably due to early reporting and prompt treatment. However, those patients who came after 7 days of onset of the disease did not regain vision even with subconjunctival and intravitreal injections.

\section{Conclusion}

SHAPU is more prevalent in the pediatric age group. It is equally prevalent among males and females. Visual acuity is severely affected by it at presentation to the hospital. The visual acuity can improve with early treatment. The effectiveness of sub-conjunctival steroid injections in the treatment for SHAPU should be further studied.

\section{Acknowledgements}

My special thanks go to Dr Sunu Dulal, Dr Indraman Maharjan, Mr Janak Bahadur Chand, Mrs Bhawani Shrestha, Mr Narayan Baral, Mr Prem Nakarmi and Mrs Shakuntala who have helped me to complete this study.

\section{References}

Albert DM, Jakobiec FA (1994). Principles and practice of ophthalmology. Ed, Vol.1, 397-405.

Byanju RN, Pradhan E, Rai NC et al (2003). Visual outcome of vitrectomy in seasonal hyperacute pan uveitis. Kath Univ Med J,1:121-123.

Malla OK (1978). Endophthalmitis probably caused by Tussock moth. In report of the proceedings of the first national seminar on prevention of blindness: 44 .

Nussenblatt RB, Palestine AG (2004). Seasonal hyperacute panuveitis. In Nussenblatt

RB, Whitcup SM, editors. Uveitis: fundamental and clinical practice, 3rd ed. St Louis, Missouri: Mosby: 311-323.

Upadhyay MP, Rai NC, Ogg JE et al (1984). Seasonal hyper acute panuveitis of unknown etiology. Ann Ophthalmol; 16:34-44.

Upadhyay MP, Rai NC, Ogg JE et al (1984). Seasonal hyperacute panuveitis. Uveitis update:257-262.

Source of support: nil. Conflict of interest: none 